\title{
A CASE OF SUB-ACUTE INTUSSUSCEPTION WITH SKIAGRAMS
}

BY

\author{
REGINALD MILLER, M.D., F.R.C.P.
}

(From the Paddington Green Children's Hospital, London.)

It is so unusual for a physician to have the opportunity of studying the radiographic appearances of an intussusception that the present case is recorded. The patient was a girl of three years old presenting an obvious

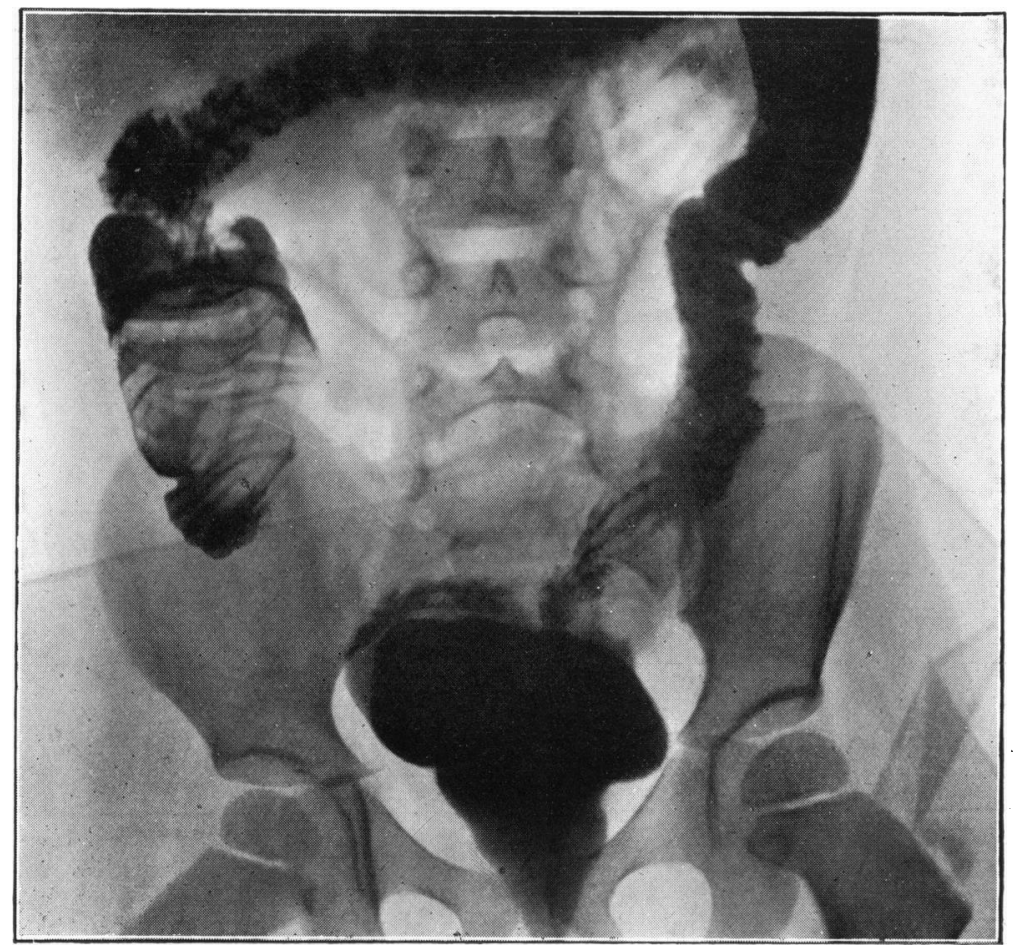

FIG. 1.-Barium enema : immediately after evacuation.

abdominal tumour which was at first regarded as a chronic intussusception. The course of the illness showed, however, that it was more properly classed as sub-acute. It produced at no time any urgent symptoms, and ultimately it disappeared either spontaneously or aided by the bowel wash-outs which were given almost daily. Laparotomy proved that the reduction of the intussusception was complete. A barium enema was given on the seventh day of disease, followed by an opaque meal two days later. 


\section{Case report.}

Florrie G., aged 3 years and 2 months, was taken with severe abdominal pain and vomiting on April 10th, 1932. She vomited all liquids and had no action of the bowels. No blood or mucus was passed per rectum. On April 13th she was admitted to hospital when she was found to show a smooth rounded mobile tumour in the left side of the abdomen at the level of the umbilicus. This was only slightly tender. No blood or mucus appeared on digital examination of the bowel. An aperient enema was given at once from which there was a large scybalous result, showing traces of blood and a little mucus. The child's general condition was quite good, and immediately after the enema the tumour could not be felt for the time being. During the next three days she was sick only once, and the bowels were open by enema. The chief symptom was that of attacks of severe colic coming on in the region of

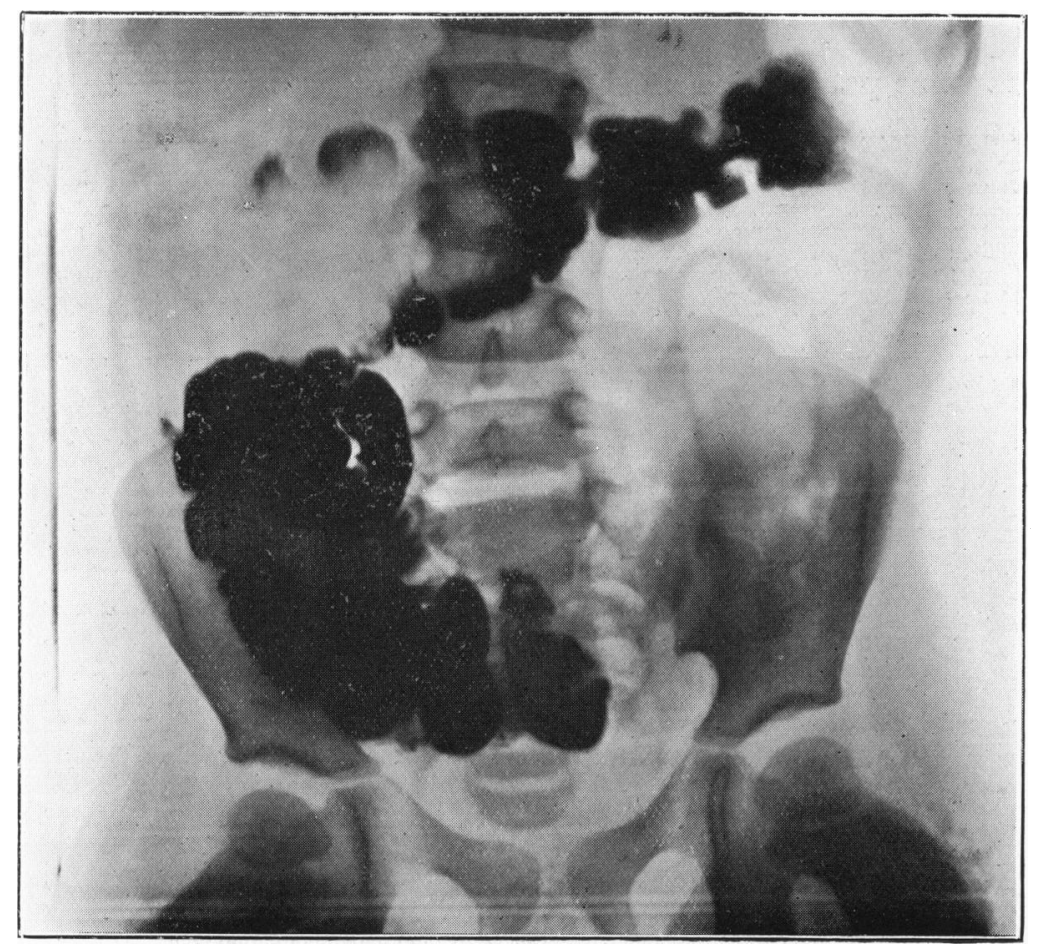

Fig. 2.-Barium meal : at 6 hours.

the tumour at irregular intervals and occurring about six times in the twenty-four hours. The tumour reappeared on the left side, and it was noticed that very shortly after the abdomen had been palpated an attack of pain would supervene. The pain lasted for a few minutes only.

I saw the child for the first time on Saturday, April 16th. She then presented an obvious tumour in the splenic region. Palpation caused the tumour to harden and gave rise to a short attack of colic. It was impossible to mistake the tumour for anything other than an intussusception, and as the child's condition on the seventh day of illness was so good I ordered an immediate examination by means of an opaque enema. The results of this (Fig. 1) clearly demonstrated the presence of an intussusception. As the child kept well an opaque meal was given on April 18th, and the films taken at 6 and 24 hours (Fig. 2 and 3) again show the 


\section{A CASE OF SUB-ACUTE INTUSSUSCEPTION}

condition well. It is noticeable that the intussusception in the skiagrams has travelled back to the right side of the patient. Seen on April 19th it was impossible to be sure of the presence of any tumour, but it was judged to be unsafe to leave the abdomen unexplored for fear of missing an incomplete reduction of the intussusception. On April 20th Mr. Shattock opened the abdomen but found that complete reduction had occurred. The ileo-cæcal region was œedematous and the adjacent glands were enlarged and soft. One of these in the ileo-cæcal angle was removed, the cæcum was stitched to the posterior abdominal wall, and a long healthy appendix was removed. Recovery was uneventful except for some bronchial catarrh.

Radiographic examinations.-Figure 1 is from a film taken immediately after the evacuation of a barium enema. It shows the apex of the intussusception just below the hepatic flexure. The barium has been

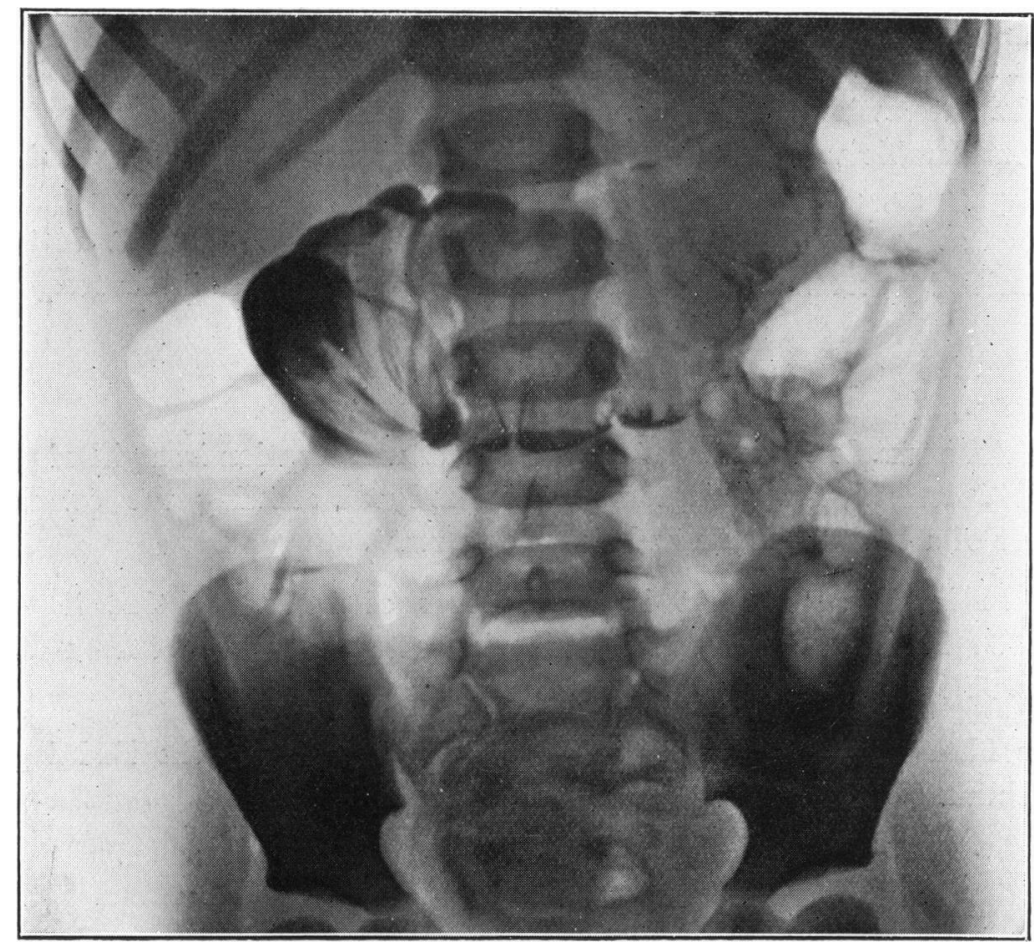

Fig. 3.-Barium meal : at 24 hours.

insinuated between the intussusceptum and the intussuscipiens, clearly showing the folds in the mucosa.

Figure 2 is from a skiagram taken six hours after an opaque meal. It shows ileal stasis and the apex of the intussusceptum at the middle of the transverse colon. Haustration is seen at the upper and lower borders of the intussuscipiens, the clear zone between being occupied by the intussusceptum. The inclusion of the barium at the upper and lower borders only is probably due to the flattening effect of the indrawn mesentery. 
Figure 3 is from a film taken twenty-four hours after ingestion of the meal. Practically the whole of the meal has been passed with the exception of a trace which perfectly outlines the apex of the intussusceptum at the extreme left of the transverse colon. The resemblance of this to the cervix and os uteri is well shown.

\section{Discussion.}

In the present case the radiographic examination cannot be said to have been of value in the diagnosis of the presence of an intussusception as the tumour gave clear evidence of that condition. It did, however, enable us to watch the gradual reduction of the intussusception, and it might, had it been tried, have been of use in demonstrating that the intussusception had been completely reduced, and so saved the child from operation.

The successful demonstration of the intussusception by an opaque enema raises the interesting question whether such means of diagnosis might not be of considerable value in doubtful and acute cases. It would seem to be worth trying as, at all events in hospital practice, it could be so quickly performed as to lead to no real waste of time. In the present instance examination by screen alone showed satisfactory evidence of intussusception.

\section{Summary and conclusions.}

1. An example of a subacute ileo-cæcal intussusception in a girl of three years is described, and skiagrams are reproduced showing the condition by means of a barium enema and an opaque meal.

2. It is suggested that in acute cases of intussusception if there is difficulty in diagnosis, screen examination after an opaque enema might give valuable information without undue waste of time.

The films were taken by Miss Frost, radiographer to the Paddington Green Children's Hospital. 